\title{
NOUVELLE
}

\section{Transdifférenciation induite}

\section{La plasticité cellulaire revisitée}

John De Vos

Institut de recherche en biothérapie, Inserm U847, Hôpital Saint-Eloi, 80 avenue A. Fliche, 34295 Montpellier, France.

john.devos@inserm.fr
> « En se réveillant un matin après des rêves agités, Gregor Samsa se retrouva, dans son lit, métamorphosé en un monstrueux insecte. Il était sur le dos, un dos aussi dur qu'une carapace, et en relevant un peu la tête, il vit, bombé, brun, cloisonné par des arceaux plus rigides, son abdomen sur le haut duquel la couverture, prête à glisser tout à fait, ne tenait plus qu'à peine... ». La nouvelle $e^{1}$ imaginée par Kafka n'est-elle qu'un cauchemar, relevant de la pure fiction? Pour se rassurer, on peut constater que les tissus de notre organisme sont dotés d'une très grande stabilité au cours du temps. Cette propriété est d'ailleurs fondamentale pour les organismes pluricellulaires que nous sommes. Grâce à elle, la peau de notre visage reste invariablement, jour après jour, de la peau, et jamais ne se trouve changée en un autre organe tel que du foie! Mais si ce phénomène est essentiel pour un fonctionnement normal de notre corps, il est aussi une limite regrettable pour les possibilités de traitement de certaines pathologies. Lorsqu'un organe est endommagé par perte cellulaire, il n'est pas possible de faire appel directement aux cellules d'un autre organe pour le régénérer. Mais en fin de compte, nous allons le voir plus loin, l'état de différenciation d'une cellule s'avère très malléable.

\section{Reprogrammation \\ par dédifférenciation \\ en cellules pluripotentes: \\ (trop?) sophistiqué \\ On savait déjà par les approches de clo- nage par transfert nucléaire - chez les}

\footnotetext{
${ }^{1}$ La Métamorphose, publiée en 1915.
}

batraciens puis les mammifères - ainsi que par la reprogrammation de cellules différenciées en cellules pluripotentes (création des iPS, induced pluripotent stem cells), qu'il était possible de remonter le temps de la différenciation et amener une cellule spécialisée vers l'état originel embryonnaire [1]. Ces travaux ont transformé la vision historique d'une différenciation cellulaire irréversible. Mais toutes ces explorations ne suivaient qu'un seul chemin : le retour en arrière, c'est-à-dire la dédifférenciation (Figure I). Au contraire, plusieurs équipes viennent de montrer qu'il est possible de transformer directement une cellule spécialisée en une autre, sans passage obligé par le stade pluripotent embryonnaire. L'équipe de Deepak Srivastava, du Gladstone Institute of Cardiovascular Disease, vient de démontrer la reprogrammation de fibroblastes cardiaques de souriceaux nouveau-nés en cardiomyocytes par l'expression, après transduction par des vecteurs rétroviraux, de trois facteurs de transcription (Gata4, Mef2c, et $T b \times 5$ ) requis pour la spécification embryonnaire du lignage musculaire cardiaque [2]. Le profil d'expression, la chromatine, les caractéristiques électrophysiologiques et mécaniques (contraction spontanée et rythmique) des cardiomyocytes induits sont similaires à ceux des cardiomyocytes isolés à partir du cœur. La démarche des auteurs a été similaire à celle qu'a utilisé Shinya Yamanaka pour identifier les 4 facteurs capables de générer des iPS, c'est-àdire débuter avec une liste élargie de facteurs de transcription puis tenter progressivement de la réduire pour trouver la combinaison minimale permet- tant une reprogrammation efficace. II s'avère que l'exclusion de certains des candidats présents dans un cocktail initial de 14 facteurs de transcriptions a grandement amélioré l'efficacité de la reprogrammation, les trois facteurs Gata4, Mef2c et Tbx5 constituant la combinaison optimale. Ces résultats ont également été reproduits à partir de fibroblastes cardiaques de souris adultes ou de fibroblastes de la queue de souris, montrant que cette reprogrammation pouvait être obtenue à tout âge et à partir de fibroblastes d'origine tissulaire variée. L'expression de la chaîne lourde alpha de la myosine, caractéristique des cellules musculaires myocardiques, apparaît dans plus de $20 \%$ des cellules de la culture et s'observe dès les premiers jours de la reprogrammation, avec un optimum 10 jours après transduction. Ces observations montrent que les cellules ciblées par la reprogrammation ne sont pas une sous-population rare particulière mais bien une fraction significative des fibroblastes. Ces rendements de reprogrammation sont très au-dessus de ceux de la reprogrammation en iPS (0,01-0,1\%). De manière remarquable, les auteurs démontrent par des techniques de marquage génétique que cette reprogrammation est directe, d'un état différencié à un autre, sans passage par un stade de cellule souche ou de progéniteur cardiaque. Un autre exemple de cette possible reprogrammation directe, publié par l'équipe de Marius Wernig dans Nature est l'observation que l'expression des facteurs de transcription Ascll, Brn2 et Mytll - eux aussi nécessaires à la spécification normale de ce lignage dans des fibroblastes de souris entraîne 


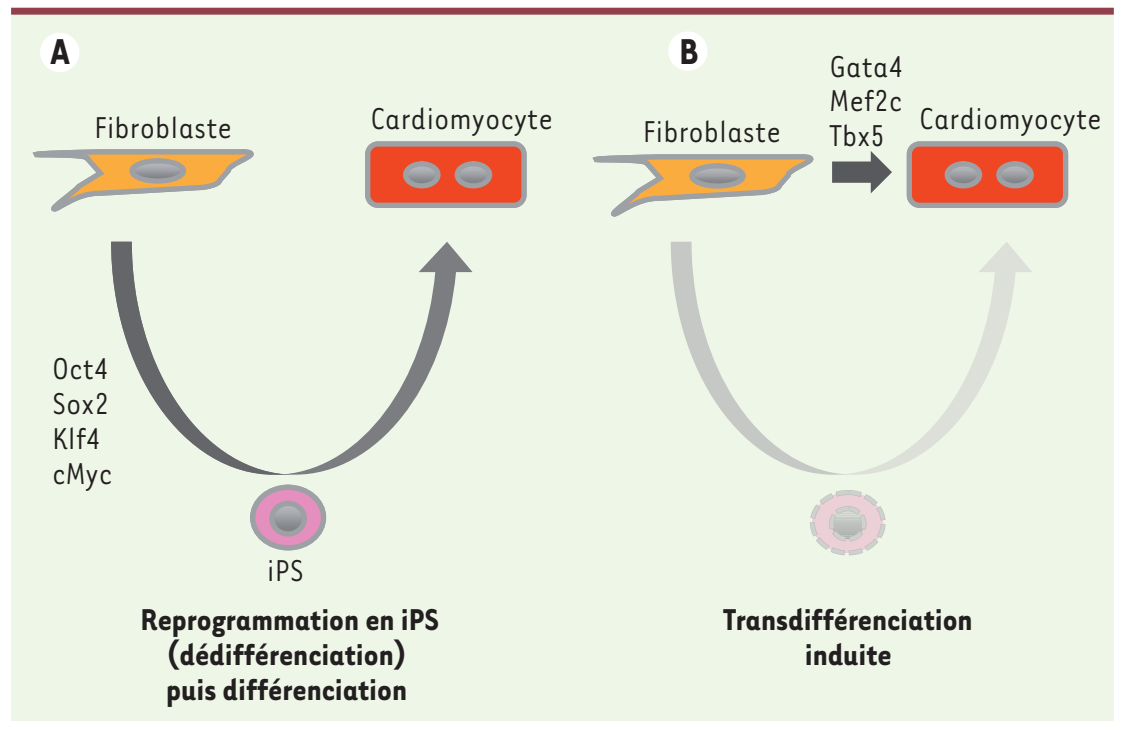

leur conversion en neurones aux potentiels d'action et synapses typiques [3]. C'est ici la démonstration de la possible conversion de cellules issues du mésoderme en cellules issues de l'ectoderme, c'est-à-dire entre types cellulaires issus de deux feuillets embryonnaires différents. Ces résultats font suite à d'autres plus anciens, démontrant par exemple la transformation d'un fibroblaste en cellule exhibant un phénotype musculaire par la surexpression du facteur de transcription MyoD [4], ou la transformation d'un lymphocyte $B$ en macrophage par la surexpression des facteurs de transcription C/EBP $\alpha$ et $\beta$, déterminants clés de l’identité du lignage granulomacrophagique [5].

\section{Reprogrammation par trans-}

différenciation : beaucoup plus direct Le passage direct d'un état de différenciation à un autre sans passage par un stade de cellule souche immature point commun aux manipulations cellulaires citées ci-dessus - est une transdifférenciation. Les cellules obtenues par transdifférenciation directe et dirigée ont déjà été baptisées et ont reçu le nom générique de cellules transdifférenciées induites (iT pour induced transdifferentiated cells) [6]. Ces derniersnés de la biothérapie ouvrent la porte à des applications cliniques, telle que la reprogrammation in vitro de fibroblastes d'un patient en cardiomyocytes ou en neurones pour réparer des organes vitaux endommagés. On peut spéculer que ces résultats seront extrapolés aux cellules sanguines, source cellulaire abondante, en permanente régénération et facilement accessible, et l'on pourrait alors obtenir des cardiomyocytes ou des neurones à partir de leucocytes. Par rapport aux cellules souches pluripotentes - cellules souches embryonnaires humaines et iPS - les cellules transdifférenciées induites pourraient offrir certains avantages: absence de formation de tératome, rapidité accrue pour générer des cellules-médicament. Mais l'application clinique la plus originale de la transdifférenciation induite pourrait être la transdifférenciation in vivo. La démonstration chez l'animal de la faisabilité de cette approche a été rapportée en 2008 par l'équipe de Douglas Melton par la reprogrammation in vivo chez la souris diabétique de cellules exocrines du pancréas en cellules endocrines produisant de l'insuline [7]. Cette conversion cellulaire a été obtenue par l'injection directe dans le pancréas d'adénovirus exprimant trois facteurs de transcription essentiels pour le développement des cellules $\beta$ de Langerhans ( $\mathrm{Ngn} 3, \mathrm{Pdxl}$ et Mafa), et s'est traduite par une amélioration de la glycémie des souris traitées
Figure 1. Reprogrammation par dédifférenciation et transdifférenciation. A. Par la technique des cellules souches pluripotentes induites (iPS) il est possible de reprogrammer des fibroblastes en cellules souches pluripotentes par l'expression des facteurs de transcription Oct4, Sox2, KIf4 et cMyc. Celles-ci peuvent ensuite être amenées à être différenciées en cardiomyocytes in vitro par des conditions de culture particulières. B. La technique de transdifférenciation induite raccourcit ce processus en convertissant directement des fibroblastes en cardiomyocytes, sans passage par un stade indifférencié, par la surexpression des facteurs de transcription Gata4, Mef2c et Tbx5 [2].

$(\rightarrow)$ [8]. À partir de toutes ces découvertes, il n'y a qu'un $(\rightarrow)$ Voir la Nouvelle de Fabrizio Thorel et Pedro L. Herrera, page 906 de ce numéro pas pour imaginer soigner l'infarctus du myocarde par l'injection de facteurs de reprogrammation directement dans la paroi cardiaque pour convertir une partie des fibroblastes de la paroi du ventricule lésé en cardiomyocytes et restaurer la contractilité myocardique.

La plasticité cellulaire avait déjà connu ses heures de gloire à la fin des années 1990. Des expériences in vivo chez l'animal avaient alors suggéré la possibilité d'une transdifférenciation. Des cellules souches d'un organe (par exemple des cellules souches hématopoïétiques) étaient injectées à une souris puis l'on traçait leur devenir. Avec surprise, l'on observait que des cellules hépatiques, musculaires ou neuronales étaient issues des cellules injectées, ce qui était en faveur de la transdifférenciation de cellules hématopoirétiques en cellules aussi différentes que des neurones. Mais ces espoirs avaient été déçus quand on avait compris que bien souvent ces observations étaient des phénomènes de fusion cellulaire, et dans les cas où l'on avait exclu la fusion, ces événements restaient rares et donc peu relevants pour la clinique ou l'étude des mécanismes de transdifférenciation [9]. La démonstration de la réelle faisabilité d'une transdifférencia- 
tion directe, dirigée, rapide et massive remet ces notions au cœur des perspectives thérapeutiques les plus passionnantes. Pour réaliser ces rêves, il conviendra de trouver une alternative aux vecteurs viraux intégratifs, par exemple par l'usage d'adénovirus ou de protéines recombinantes. II faudra également s'assurer que la transdifférenciation s'effectue dans la bonne direction, celle de l'organe à soigner et non d'un autre organe, voire en des cellules n'existant pas naturellement, sans quoi la réalisation de la plasticité cellulaire pourrait transformer le rêve de médecine régénérative en cauchemar de Kafka! $\diamond$
Induced transdifferentiation : revisiting cellular plasticity

\section{CONFLIT D'INTÉRÊTS}

Les auteurs déclarent n'avoir aucun conflit d'intérêts concernant les données publiées dans cet article.

\section{RéFÉRENCES}

1. Coulombel L. Reprogrammation nucléaire d'une cellule différenciée : on efface tout et on recommence. Med Sci (Paris) $2007 ; 23: 667-70$.

2. leda M, Fu JD, Delgado-Olguin P, et al. Direct reprogramming of fibroblasts into functional cardiomyocytes by defined factors. Cell 2010 ; 142 : 375-86.

3. Vierbuchen T, Ostermeier A, Pang ZP, et al. Direct conversion of fibroblasts to functional neurons by defined factors. Nature $2010 ; 463$ : 1035-41.

\section{NOUVELLE}

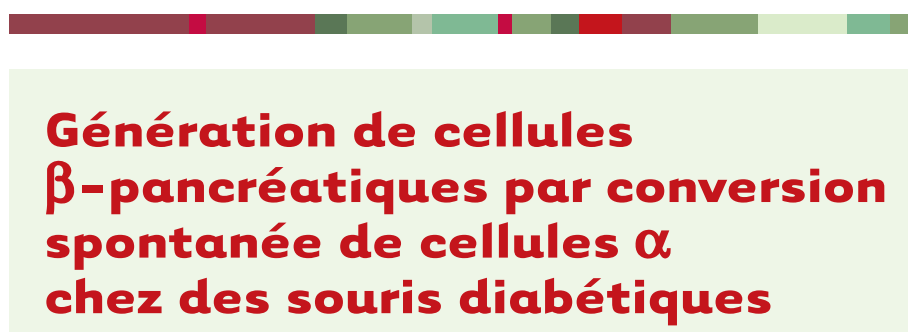

Fabrizio Thorel, Pedro L. Herrera

Fonctions endocrines

\section{et exocrines du pancréas}

Le pancréas est une glande qui remplit deux fonctions métaboliques importantes. La première est la production, par les cellules acinaires pancréatiques, des enzymes digestives nécessaires à la dégradation des aliments. Ces enzymes sont excrétées et acheminées au travers d'un réseau de canaux jusqu'à l'intestin, où les aliments seront digérés et absorbés. Les cellules acinaires et canalaires constituent la partie exocrine du pancréas et représentent environ $99 \%$ de toutes les cellules de cet organe.

La seconde fonction du pancréas est la synthèse des hormones responsables de la régulation de l'utilisation du sucre issu de la digestion des aliments et donc du contrôle de la glycémie (taux de sucre dans la circulation sanguine). Cette régulation permet de contrebalancer les épisodes d'hyperglycémie après les prises alimentaires et d'augmenter la glycémie à la suite du jeûne. La production d'hormones est assurée par la partie endocrine du pancréas, qui est organisée en groupes de cellules appelés îlots de Langerhans. Ceux-ci ne représentent que $1 \%$ du poids de l'organe. Les cellules $\alpha$, $\beta, \delta$ et PP synthétisent, respectivement, le glucagon, l'insuline, la somatostatine et le polypeptide pancréatique. Elles constituent les différents types cellulaires endocriniens matures des îlots pancréatiques adultes [1].

Le besoin de nouvelles approches thérapeutiques anti-diabétiques Les cellules $\beta$ ont une grande longévité et, par conséquent, prolifèrent peu au cours de la vie d'un individu. Lors de circonstances où la demande en insuline est accrue, comme en cas d'obésité ou pendant la grossesse, la taille et le nombre des cellules $\beta$ peuvent
4. Tapscott SJ, Davis RL, Thayer MJ, et al. MyoDl: a nuclear phosphoprotein requiring a Myc homology region to convert fibroblasts to myoblasts. Science $1988 ; 242: 405-11$.

5. Xie H, Ye M, Feng R, Graf T. Stepwise reprogramming of $B$ cells into macrophages. Cell $2004 ; 117$ : 663-76.

6. Masip M, Veiga A, Izpisua JC, Simon C. Reprogramming with defined factors: from induced pluripotency to induced transdifferentiation. Mol Hum Reprod 2010, sous presse

7. Zhou Q, Brown J, Kanarek A, et al. In vivo reprogramming of adult pancreatic exocrine cells to beta-cells. Nature $2008 ; 455: 627-32$.

8. Collombat P, Mansouri A. Pax4 transdifferentiates glucagon-secreting alpha cells to insulin-secreting beta endocrine pancreatic cells. Med Sci (Paris) 2009; $25: 763-5$.

9. Coulombel L. Cellules souches tissulaires adultes: seing is not being. Med Sci (Paris) 2003; $19: 683-94$.
Département de physiologie cellulaire et métabolisme, Faculté de médecine, Université de Genève, 1 , rue Michel-Servet, CH 1211 Genève-4, Suisse. pedro.herrera@unige.ch

augmenter, respectivement via une augmentation de la production d'insuline et la stimulation de la division cellulaire. Un déficit en cellules $\beta$, ou l'incapacité de celles-ci à produire suffisamment d'insuline, conduit à une augmentation soutenue de la glycémie se traduisant par une production élevée d'urine, c'est-à-dire un diabète. Dans le diabète de type 1 , maladie auto-immune qui représente la forme la plus courante de la maladie chez l'enfant, les cellules $\beta$ sont éliminées complètement par le système immunitaire. Par conséquent, ces patients doivent recevoir des injections quotidiennes d'insuline pendant toute leur vie. Cependant, cette stratégie thérapeutique ne permet pas un contrôle précis de la glycémie et n'évite pas les effets nocifs sur les vaisseaux sanguins qui peuvent conduire à l'insuffisance rénale, la cécité ou la gangrène. Ces complications justifient le déve- 